\title{
Comparison of the serological tests ICT and ELISA for the diagnosis of alveolar echinococcosis in France
}

\author{
Jenny Knapp ${ }^{1,2, *}$, Yasuhito Sako ${ }^{3}$, Frédéric Grenouillet ${ }^{1,2,4}$, Solange Bresson-Hadni ${ }^{2,5}$, \\ Carine Richou $^{2,5}$, Houssein Gbaguidi-Haore ${ }^{1,6}$, Akira Ito ${ }^{3}$, and Laurence Millon ${ }^{1,2,4}$ \\ ${ }^{1}$ Laboratory of Chrono-environnement, UMR/CNRS 6249, Faculty of Medicine and Pharmacy, Besançon, France \\ 2 WHO Collaborating Centre for prevention and treatment of human echinococcosis, Besançon, France \\ 3 Department of Parasitology, Asahikawa Medical University, Asahikawa, Hokkaido, Japan \\ ${ }^{4}$ Laboratory of Parasitology-Mycology, University Hospital of Besançon, France \\ 5 Department of Hepatology, University Hospital of Besançon, France \\ 6 Laboratory of Hospital Hygiene, University Hospital of Besançon, France
}

Received 18 October 2013, Accepted 4 July 2014, Published online 25 July 2014

\begin{abstract}
Serological diagnosis of alveolar echinococcosis (AE) is a key element for efficient patient treatment management. A rapid immunochromatography test kit (ICT) using the recombinant Em18 antigen (rEm18) was recently developed. The aim of our study was to assess this test on a panel of sera from French patients with alveolar echinococcosis and control patients. In a blind test, a total of 112 serum samples were tested including samples of AE $(n=30)$, cystic echinococcosis [CE] $(n=15)$, and polycystic echinococcosis [PE] $(n=1)$. For the comparison, 66 sera from patients with hepatocarcinoma, fascioliasis, toxocariasis, Caroli's disease, or autoimmune chronic active hepatitis were used. The diagnostic test sets we used were the rEm18-ICT and two validated ELISAs with rEm18 and Em2Em18 antigens, respectively. For the ICT, 27/30 sera from AE patients, 4/15 sera from CE patients and the PE patient serum were positive. One serum from the control panel (toxocariasis) was positive for the ICT. The rEm18-ICT sensitivity (90.0\%) and specificity (92.7\%) for detection of Em18-specific antibodies confirmed it as a relevant tool for AE diagnosis. The rEm18-ELISA had a sensitivity of $86.7 \%$ and specificity of $91.5 \%$, and the Em2-Em18-ELISA had a sensitivity of $96.7 \%$ and specificity of $87.8 \%$. However, when AE patient sera are recorded as weak in intensity with the ICT, we recommend a double reading and use of a reference sample if the ICT is used for patient follow-up.
\end{abstract}

Key words: alveolar echinococcosis, diagnosis, rEm18, immunochromatography, rapid test, rEm18-ELISA and Em2-Em18-ELISA tests.

Résumé - Comparaison des tests sérologiques ICT et ELISA pour le diagnostic de l'échinococcose alvéolaire en France. Le diagnostic sérologique de l'échinococcose alvéolaire (EA) est un point fondamental pour assurer l'organisation $\mathrm{du}$ traitement $\mathrm{du}$ patient. Un test rapide immuno-chromatographique (ICT), utilisant l'antigène recombinant Em18 a été récemment développé. Le but de cette étude était d'évaluer ce test sur un panel de sérums de patients français présentant une échinococcose alvéolaire et sur des patients de contrôle. Lors d'un test réalisé en aveugle, un total de 112 sérums a été testé, regroupant des échantillons d'EA $(n=30)$, d'échinococcoses kystiques (EK) $(n=15)$ et d'échinococcose polykystique (EP) $(n=1)$. Pour comparaison, 66 patients présentant un hépatocarcinome, une distomatose, une toxocarose, une maladie de Caroli ou une hépatite active chronique autoimmune ont été utilisés. Les tests diagnostiques employés étaient l'ICT rEm18 et deux tests ELISA validés utilisant respectivement les antigènes $\mathrm{rEm} 18$ et Em2-Em18. Avec l'ICT, 27/30 sérums de patients EA, 4/15 EK et le patient EP étaient positifs. Un sérum du panel contrôle (toxocarose) présentait un ICT positif. Pour le test ICT rEm18, la sensibilité $(90,0 \%)$ et la spécificité $(92,7 \%)$ pour la détection de l'antigène Em18 confirme que ce test est un outil fiable pour le diagnostic d'EA. L'ELISA rEm18 présentait une sensibilité de 86,7\% et une spécificité de 91,5\%, et l'ELISA Em2-Em18 une sensibilité de $96,7 \%$ et une spécificité de $87,8 \%$. Néanmoins lorsque des sérums de patients présentent une bande test d'intensité faible pour l'ICT nous recommandons une double lecture et l'emploi d'un échantillon de référence pour une utilisation en suivi.

\footnotetext{
*Corresponding author: jenny.knapp@univ-fcomte.fr

Innovation for the Management of Echinococcosis.

Invited editors: Dominique A. Vuitton, Laurence Millon, Bruno Gottstein and Patrick Giraudoux
}

This is an Open Access article distributed under the terms of the Creative Commons Attribution License (http://creativecommons.org/licenses/by/4.0), which permits unrestricted use, distribution, and reproduction in any medium, provided the original work is properly cited. 


\section{Introduction}

In rodents and humans, alveolar echinococcosis (AE) appears as a tumor-like lesion and is caused by accidental ingestion of eggs of the cestode Echinococcus multilocularis, a fox tapeworm. The adult worm produces eggs which are released into the environment with fox feces. The parasite is widely present in the Northern Hemisphere in countries such as China, where the estimated prevalence ranges from $0.2 \%$ to $9 \%$ in 12 regions [22]. The parasite is also present in temperate Europe, where a total of 559 human cases have been identified in nine countries [9, 14]. A recent increase in reported cases of human AE and of E. multilocularis in wild animals has been observed not only in historically endemic regions in Europe [6, 20], but also in new endemic regions [5, 23]. In France, from 1982 to 2009, 8 to 29 new cases per year, mostly in the Northeast, were identified by the FrancEchino network [12]. The exposure to eggs is likely due to repeated contact with wild or domestic carnivores such as foxes, dogs, and cats [21], consumption of wild berries or raw vegetables growing close to the ground, and agricultural activities [3]. The main AE symptoms are abdominal pain, asthenia, and hepatomegaly. Generally, the first symptoms appear 5-15 years after contamination $[1,3]$. Diagnosis is often made based on images obtained by ultrasound, computerized tomography, or magnetic resonance imaging [4]. Immunodiagnosis tests, e.g., the enzyme-linked immunosorbent assay (ELISA) using rEm18 (rEm18-ELISA) [18] or rEm18 plus the native Em2 antigen purified from E. multilocularis larvae (Em2-Em18-ELISA) (Bordier Affinity, Crissier, Switzerland), are currently being used in laboratories. Indirect hemagglutination (IHA) (Hydatidose Fumouze kit, Fumouze Diagnostics, Levallois-Perret, France) is one of the low-cost screening techniques [11], and the Western blot technique (WB) (LDBIO Diagnostics, Lyon, France), using a whole E. multilocularis larval antigen, is the confirmation technique for species diagnosis $[4,16]$.

In 2003, Xiao et al. demonstrated the specificity of rEm18 for $\mathrm{AE}$ diagnosis using serum samples from patients with other parasitic infections and hepatic diseases [24]. In addition, they demonstrated that measurement of rEm18-specific antibodies can give information on parasite status after implementation of treatment [13], because antibody response against this recombinant antigen reflects the activity of the parasite. Recently, an immunochromatography test (ICT) using the rEm18 antigen was developed [17] and a sensitivity of $94 \%$ and a specificity of 95.4\% were found for AE sera. This kit is commercially available now (ADAMU-AE kit, ICST Co. Ltd., Saitama, Japan).

The main aims of our study were to assess the reliability of the ICT results in the detection of AE cases using a panel of French sera, by comparing the ICT with ELISA tests, which are validated and routinely used in laboratories, and to assess rEm18-ICT reproducibility on different batches of kits.

\section{Materials and methods}

\section{Serum samples}

A total of 112 serum samples were collected from patients. Sera were received from 1987 to 2010 at the Parasitology
Department (University Hospital, Besançon, France) for diagnosis of Echinococcus and other pathologies. The Echinococcus panel (46 samples) was composed of 30 AE (29 with liver lesions as a primary focus, and one with a lung lesion), $15 \mathrm{CE}$, and 1 polycystic echinococcosis (PE) (due to E. vogeli infection) [15]. Only AE cases based on the consensual criteria established by Brunetti et al. were included. Diagnosis had been carried out by clinical findings, imaging techniques, serology with Western Blot (LDBIO, Diagnostics, Lyon, France), specific PCR and/or histology [4]. The sera of AE patients were sampled before any parasitostatic treatment. The control serum collection (66 samples) was composed of 13 toxocariasis, 13 hepatocellular carcinoma, 8 fascioliasis, 7 autoimmune systemic diseases with high levels of circulating rheumatoid factors, 7 Caroli's disease, 5 autoimmune chronic active hepatitis, and 13 other pathologies involving the liver, i.e., liver cysts $(n=3)$, biliary cirrhosis $(n=3)$, angioma $(n=2)$, metastasis of breast carcinoma $(n=1)$, cystadenocarcinoma $(n=1)$, bacterial abscess $(n=1)$, bile duct carcinoma $(n=1)$, and neurocysticercosis $(n=1)$. For the fascioliasis and toxocariasis cases, the serology (specific serum antibodies assessed by highly sensitive serological tests, and confirmed by WB, a separate high specificity serological test) was classified as positive by clinical and epidemiological history (LDBIO Diagnosis, Lyon, France).

\section{rEm18 Immunochromatography Test}

The rEm18-ICT was based on the detection of antibodies against the recombinant Em18 antigen (rEm18) [18]. The antigen and anti-goat immunoglobulin $\mathrm{G}(\mathrm{IgG})$ as test and control lines, respectively, were sprayed onto a nitrocellulose membrane, and placed in a plastic device [17]. For the assay, first, $10 \mu \mathrm{L}$ of the serum sample were mixed in a tube with $20 \mu \mathrm{L}$ of a serum dilution buffer containing $0.1 \mathrm{mg} / \mathrm{mL}$ alkaline phosphatase-conjugated goat anti-human IgG antibody (DAKO, Tokyo, Japan). This mixed serum sample was then applied onto the sample window of the plastic device, and within $30 \mathrm{~s}$, $200 \mu \mathrm{L}$ of the substrate solution was loaded and the result was determined after $30 \mathrm{~min}$. For color development, 5-bromo-4-chloro-3-indolyl-phosphate was used. A sample was considered positive if two color lines were present after $30 \mathrm{~min}$, indicating the presence of the specific anti-recEm18 antibodies (test line: rEm18), and if the control line (anti-goat $\mathrm{IgG}$ ) was visible (indicating that the test was performed correctly). Because the ICT is a manual test, the cut-off has to be determined by visual observation. In order to obtain objective ICT results for this study, two different persons independently inspected the bands that appeared on the ICT device. After that, for the positive tests, an index intensity was calculated to enable comparison of the test results. For all samples, a picture was taken using a ChemiDoc apparatus (Bio-Rad laboratories, Hercules, CA, USA) and the intensities of the control and test bands and of the background membrane were recorded, after exposure to Epi-white light (Quantity One 4.6.5 software). The relative intensity index was calculated so that batches used for the same sera could be compared. The index intensity calculation in our study differs from that of Sako et al. [19] because there is no immunochromato-reader in the 
Table 1. Results for ELISA tests rEm18 and Em2-Em18, and rEm18-ICT for AE (alveolar echinococcosis), CE and PE (cystic and polycystic echinococcosis), and other pathologies. Percentages of sensitivity, specificity, and the performance index for each test are shown.

\begin{tabular}{|c|c|c|c|c|}
\hline & \multirow[t]{2}{*}{ No. of samples } & \multicolumn{3}{|c|}{ No. of seropositive samples for the three tests } \\
\hline & & rEm18-ICT & rEm18-ELISA & Em2-Em18-ELISA \\
\hline$\overline{\mathrm{AE}}$ & 30 & 27 & 26 & 29 \\
\hline $\mathrm{CE} / \mathrm{PE}$ & 16 & 5 & 4 & 5 \\
\hline Other & 66 & 1 & 3 & 5 \\
\hline Sensitivity, $\%$ & & 90.0 & 86.7 & 96.7 \\
\hline$(95 \% \mathrm{CI})$ & & $(78.4-96.1)$ & $(74.4-93.9)$ & $(85.8-99.4)$ \\
\hline Specificity, \% & & 92.7 & 91.5 & 87.8 \\
\hline$(95 \% \mathrm{CI})$ & & $(88.4-94.9)$ & $(87.0-94.1)$ & $(83.8-88.8)$ \\
\hline Youden index & & 0.83 & 0.78 & 0.85 \\
\hline$(95 \% \mathrm{CI})$ & & $(0.67-0.91)$ & $(0.61-0.88)$ & $(0.70-0.88)$ \\
\hline Accuracy, \% & & 92.0 & 90.2 & 90.2 \\
\hline$(95 \% \mathrm{CI})$ & & $(85.7-95.2)$ & $(83.6-94.1)$ & $(84.4-91.6)$ \\
\hline Positive likelihood ratio $(\mathrm{LR}+)$ & & 12.3 & 10.2 & 7.9 \\
\hline$(95 \% \mathrm{CI})$ & & $(6.7-18.9)$ & $(5.7-16.0)$ & $(5.3-8.9)$ \\
\hline Negative likelihood ratio (LR-) & & 0.11 & 0.15 & 0.04 \\
\hline$(95 \% \mathrm{CI})$ & & $(0.04-0.25)$ & $(0.06-0.30)$ & $(0.01-0.17)$ \\
\hline Diagnostic odds ratio & & 114.0 & 69.6 & 208.8 \\
\hline$(95 \% \mathrm{CI})$ & & $(27.7-461.2)$ & $(19.4-248.5)$ & $(31.5-1314.4)$ \\
\hline
\end{tabular}

Chrono-Environnement Laboratory at the Faculty of Medicine and Pharmacy, Besançon, France.

The background membrane intensity was subtracted from the test and control band intensities, yielding the following formula:

Index intensity $=$ (test band intensity - background intensity) /

(control band intensity - background intensity).

\section{Em2-Em18-ELISA and rEm18-ELISA}

The Em2-Em18-ELISA detects IgG antibodies in human serum against Em2 and Em18 antigens (Bordier Affinity, Crissier, Switzerland). IgG antibodies were detected with a protein A-alkaline phosphatase conjugate. Microtitration was assessed by ELISA on an Evolis microplate automaton (BioRad Laboratories, Hercules, CA, USA). The cut-off value calculation was established as recommended by the manufacturer, and the "home index" was considered as a positive value when $60 \%$ of the recommended index value was attained. Thus, a high sensitivity level was achieved, while maintaining good specificity.

The rEm18-ELISA was performed as previously described [18]. Optical density (OD) was measured and the cut-off value was 0.12 . This cut-off value was calculated from the OD values from 40 negative controls (healthy people with no clinical features of AE). The average was calculated and 4 standard deviations were added to provide the cut-off value. Serum samples were sent to Japan and analyzed in a blind test at the Department of Parasitology, Asahikawa Medical University, Hokkaido, Japan.

\section{Statistical analysis}

Overall reproducibility was tested with the Friedman test, and pairwise comparisons were tested with the Wilcoxon signed-rank test. Indicators of diagnostic test performance were: sensitivity (Se), specificity (Sp), the Youden index $(\mathrm{YI}=\mathrm{Se}+\mathrm{Sp}-1)$, to assess the efficiency of the test (negative value for an inefficient test, positive value for a reliable test), and accuracy (proportion of patients correctly classified). Positive and negative likelihood ratios (LR+ and LR-), which respectively describe the discriminatory abilities of positive and negative test results, were calculated, with a $95 \%$ confidence interval $(95 \% \mathrm{CI})$. A LR+ value above 10 and a $\mathrm{LR}-$ value below 0.1 were used to confirm the relevance of the diagnostic tool [8]. The diagnostic odds ratio $(\mathrm{DOR}=\mathrm{LR}+/ \mathrm{LR}-)$ was also determined. The DOR can be used on its own to indicate a test's discriminatory performance [10]. All analyses were twotailed, and a $p$-value of less than 0.05 was considered significant. The software package Stata 10.0 (StataCorp LP, College Station, TX, USA) was used for the analysis.

\section{Results}

\section{rEm18-ICT: sensitivity, specificity and reproducibility}

Twenty-seven out of 30 AE sera were positive with rEm18ICT as observed visually by independent double reading, demonstrating a sensitivity of $90.0 \%$ (Table 1 and Fig. 1). Four of the $15 \mathrm{CE}$ cases tested were positive for the ICT; the PE case was also positive. One of the 66 control sera was positive ( 1 toxocariasis, in Fig. 1: TOX1).

Thirty-three sera out of the 112 tested were ranked positive by rEm18-ICT with 27 specific diagnoses, with a specificity of 92.7 (Table 1).

The reproducibility of band intensities was assessed on 10 different sera tested with three different batches of kits (Figs. 2A-2B). A significant difference was observed between batch 3 and the two others $(p=0.025)$. A significant difference 


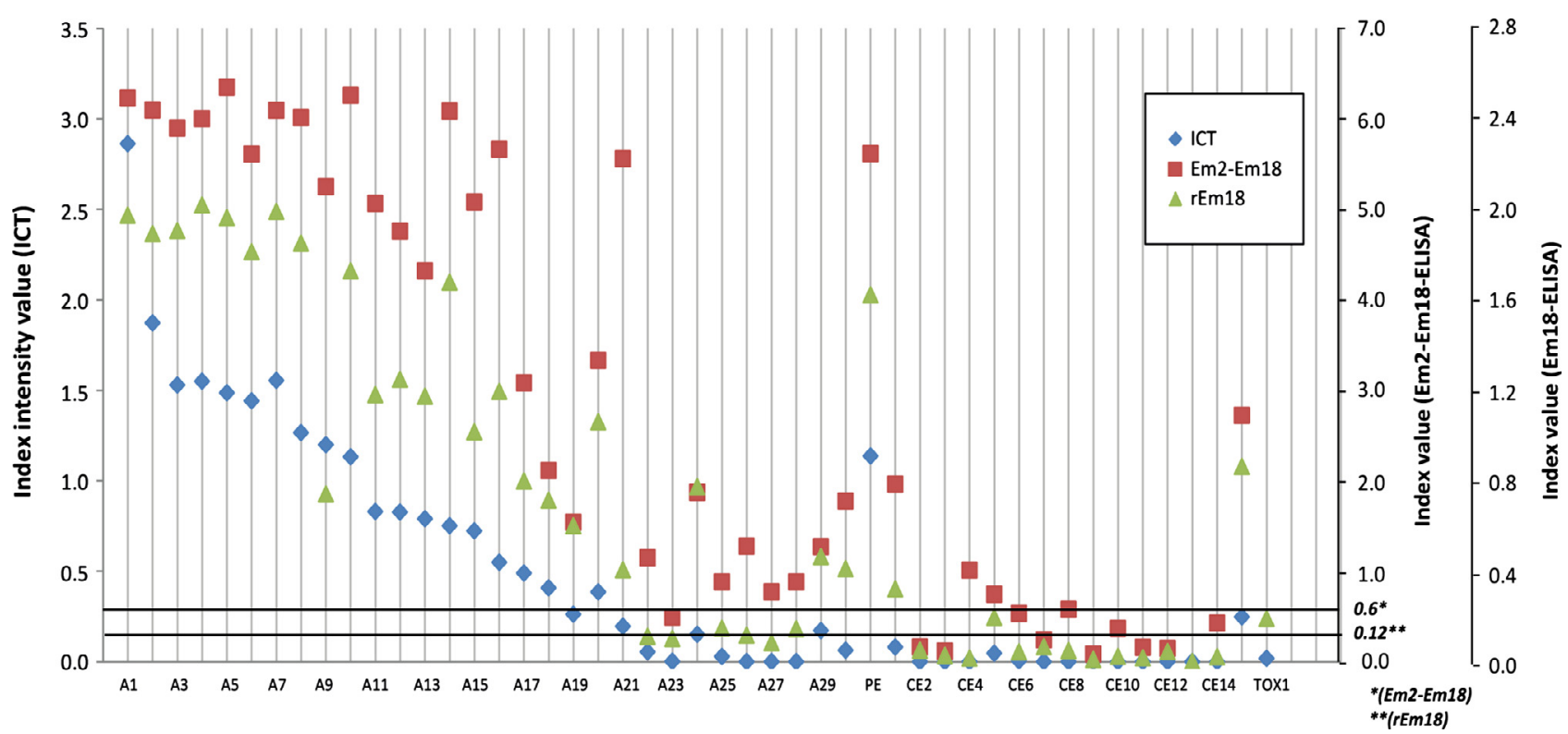

Figure 1. Immunological response to E. multilocularis antigens. Comparison of rEm18-ICT with ELISA rEm18 (OD values) and Em2-Em18 (index values) for AE (alveolar echinococcosis), CE (cystic echinococcosis), PE (polycystic echinococcosis), and toxocariasis (TOX1) cases. The thin dotted line represents the Em2-Em18 index threshold and the thick dotted line represents the rEm18 OD threshold; *Em2-Em18 threshold index, **rEm18 threshold OD value.
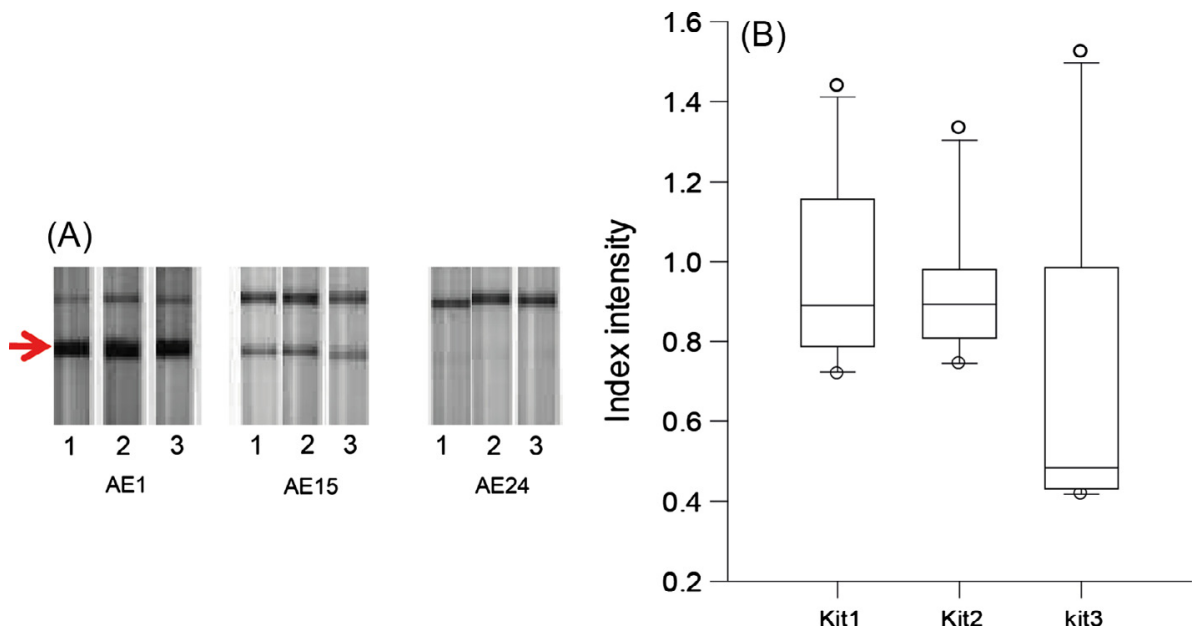

Figure 2. Test of reproducibility of rEm18-ICT using sera from 10 French AE (alveolar echinococcosis) patients, with three batches of kits (numbers 1, 2, and 3). (A) Strong, middle, and weak signals are shown for patients AE1, AE15, and AE24. The arrow shows the test band that indicates a positive test. (B) Index intensity values of three batches of kits; $p<0.05$.

was observed between batches No. 3 and No. $1(p=0.0093)$ and between No. 3 and No. $2(p=0.0125)$.

\section{ICT versus ELISAs}

Thirty-three cases, including $26 \mathrm{AE}, 3 \mathrm{CE}, 1 \mathrm{PE}$, and 3 other diseases (liver cyst, toxocariasis [TOX1], and hepatocellular carcinoma), similar to the rEm18-ICT results (Table 1 and Fig. 1), were ranked seropositive by rEm18-ELISA, and 39 cases, including 29 AE, 4 CE, 1 PE, and 5 other diagnoses (liver metastasis, breast carcinoma, hepatocellular carcinoma, two cases of fascioliasis, and autoimmune chronic active hepatitis) were ranked seropositive by Em2-Em18-ELISA. There were no differences in diagnostic performance among the tests evaluated.

No significant differences were found in sensitivity and specificity among the methods used. (Fig. 1 and Table 1).

Five results were discordant between $\mathrm{rEm} 18$-ICT and the ELISAs for the diagnosis of AE. For one case, the ICT was weakly positive, whereas the two ELISAs were negative (AE23 in Fig. 1). For two cases, rEm18-ICT and rEm18-ELISA gave a negative result, and Em2-Em18-ELISA was positive (AE26 and AE27). For one case, rEm18-ELISA was negative, and rEm18-ICT and Em2-Em18-ELISA were positive (AE22). For the fifth case, the ICT was negative and the ELISAs were 
positive (AE28). The toxocariasis serum TOX1, positive with rEm18-ICT, was also positive with rEm18-ELISA, but not with Em2-Em18-ELISA.

For the ICT, the values of the LRs $(\mathrm{LR}+=12.3$ and $\mathrm{LR}-=0.11)$ confirmed the relevance of this diagnostic tool. In addition, the DOR, Youden index, and accuracy emphasized the relevance of the ICT for AE diagnosis and the reliability of its results compared with the other tests (Table 1), because no significant difference was observed.

\section{Discussion}

Previous evaluations have revealed that the rEm18-ICT is a reliable and rapid test, applicable for $\mathrm{AE}$ diagnosis at first screening. It is worth noting that in the field, it is particularly handy because the kit comes in a plastic case. Our study focused on the usefulness of the ICT for routine serological diagnosis. The ICT provided good scores for specificity, sensitivity, the Youden index, accuracy and the LRs. In addition, DOR analysis confirmed that the ICT performs similarly to validated tests, i.e., ELISA. However, our results suggest that a combination of different serological tests seems to be necessary for an accurate diagnosis, especially for low immunological responses (Fig. 2A). The sera were tested in order to assess the usefulness of the ICT in comparison with ELISAs for differential diagnoses in the laboratory. Because sera from patients with different pathologies, and not from healthy individuals, were used in this study, the diagnostic parameters provided by our analysis, including sensitivity and specificity, could not be generalized. Our focus was on the practicability of the test. The Em2-Em18-ELISA test provided the highest sensitivity, even if the specificity was not optimal. Routinely, the immunoblotting test is used to compensate for this deficiency. Detection of antibodies against the Em18 antigen has been demonstrated as a good marker for the study of Echinococcus activity in humans [13]. The rEm18-ICT and rEm18-ELISA have been proved useful in the laboratory to check therapy efficiency [19]. Based on serological follow-up and imaging (especially PET scan) [7], treatment can be adjusted on a case-by-case basis. Recent reports have indicated that, in some AE patients under long-term benzimidazole therapy, a parasitocidal effect may be observed [7]. If the activity of the parasite is indirectly checked by the immune response of the patient, it might be an indication that treatment should be ended.

The performance of the ELISA test combining Em2 and Em18 antigens was compared with rEm18-ELISA in a long patient surveillance study [2]. Both tests showed correlated results throughout the patient survey, with variations in immune activity paralleling curative or recurrence events after surgery.

For a manual test, because judgements are made based on visual observation, reproducibility is an absolute necessity, especially in the case of a weak immune response. Our findings on reproducibility indicated that slight differences in intensity can appear among ICT batches for the same serum sample presenting a weak immune response. When the rEm18-ICT is used in $\mathrm{AE}$ patient first screening tests or follow-up, two different operators must perform the reading and a reference sample should be included when the ICT is done. In our study the variability among the ELISA kit batches we used was also taken into account by including a reference sample in each ELISA series, and then calculating the index value based on this sample, as recommended by the manufacturer.

In conclusion, our findings demonstrate that the rEm18-ICT is a simple, reliable, and easy-to-use tool in $\mathrm{AE}$ diagnosis, requiring a minimum of equipment and time, especially for the first screening.

Acknowledgements. We are very grateful to M.-P. Faivre, F. Grenouillet, and S. Itoh for technical support and to S. Rocchi for the double reading of the ICT tests. This study was supported by the CNRS/JSPS Bilateral Research Project and the International Research Fund from the JSPS to Pr. A. Ito (21256003). We are grateful to Lois Rose for editorial assistance. We dedicate this joint paper to Mrs. Hikari Ito.

\section{References}

1. Ammann R, Eckert J. 1996. Cestodes. Echinococcus, Gastroenterology Clinics of North America, 25, 655-689.

2. Bresson-Hadni S, Blagosklonov O, Knapp J, Grenouillet F, Sako Y, Delabrousse E, Brientini MP, Richou C, Minello A, Antonino AT, Gillet M, Ito A, Mantion GA, Vuitton DA. 2011. Should possible recurrence of disease contraindicate liver transplantation in patients with end-stage alveolar echinococcosis? A 20-year follow-up study. Liver Transplantation, 17, 855-865.

3. Bresson-Hadni S, Piarroux R, Bartholomot B, Miguet J, Mantion GA, Vuitton DA. 2005. Echinococcose alvéolaire Alveolar echinococcosis. Encyclopédie Médico-Chirurgicale Hépato-Gastroentérologie, 2, 86-104.

4. Brunetti E, Kern P, Vuitton DA, WHO-IWGE W.P.f.t.. 2010. Expert consensus for the diagnosis and treatment of cystic and alveolar echinococcosis in humans. Acta Tropica, 114, 1-16.

5. Bruzinskaite R, Marcinkute A, Strupas K, Sokolovas V, Deplazes P, Mathis A, Eddi C, Sarkūnas M. 2007. Alveolar echinococcosis Lithuania. Emerging Infectious Diseases, 13, $1618-1619$

6. Combes B, Comte S, Raton V, Raoul F, Boué F, Umhang G, Favier S, Dunoyer C, Woronoff N, Giraudoux P. 2012. Westward wide spread of Echinococcus multilocularis in foxes in France. Emerging Infectious Diseases, 188, 301-305.

7. Crouzet J, Grenouillet F, Delabrousse E, Blagosklonov O, Thevenot T, Di Martino V, Piarroux R, Mantion GA, BressonHadni S. 2010. Personalized management of patients with inoperable alveolar echinococcosis undergoing treatment with albendazole: usefulness of positron-emission-tomography combined with serological and computed tomography follow-up. Clinical Microbiology and Infection, 16, 788-791.

8. Deeks JJ, Altman DG. 2004. Diagnostic tests 4: likelihood ratios. British Medical Journal, 329, 168-169.

9. Eckert J, Schantz P, Gasser R, Torgerson P, Bessonov A, Movsessian S, Thakur A, Grimm F, Nikogossian M. 2001. Geographical distribution and prevalence. Public Health Problem of Global, 1, 100-142.

10. Glas AS, Lijmer JG, Prins MH, Bonsel GJ, Bossuyt PM. 2003. The diagnostic odds ratio: a single indicator of test performance. Journal of Clinical Epidemiology, 56, 1129-1135. 
11. Gottstein B, Jacquier P, Bresson-Hadni S, Eckert J. 1993. Improved primary immunodiagnosis of alveolar echinococcosis in humans by an enzyme-linked immunosorbent assay using the Em2plus antigen. Journal of Clinical Microbiology, 31, 373-376.

12. Grenouillet F, Knapp J, Millon L, Raton V, Richou C, Piarroux M, Piarroux R, Mantion G, Vuitton D, Bresson-Hadni S. 2010. L'échinococcose alvéolaire humaine en France en 2010/Human alveolar echinococcosis in France update 2010. Bulletin Épidémiologique Hebdomadaire, Vol. Hors-série 14 septembre, 2010, 24-25.

13. Ishikawa $\mathrm{Y}$, Sako $\mathrm{Y}$, Itoh $\mathrm{S}$, Ohtake $\mathrm{T}$, Kohgo $\mathrm{Y}$, Matsuno $\mathrm{T}$, Ohsaki Y, Miyokawa N, Nakao M, Nakaya K, Ito A. 2009. Serological monitoring of progression of alveolar echinococcosis with multiorgan involvement by use of recombinant Em18. Journal of Clinical Microbiology, 47, 3191-3196.

14. Kern P, Bardonnet K, Renner E, Auer H, Pawlowski Z, Ammann RW, Vuitton DA, Registry EE. 2003. European echinococcosis registry: human alveolar echinococcosis Europe 1982-2000. Emerging Infectious Diseases, 9, 343-349.

15. Knapp J, Chirica M, Simonnet C, Grenouillet F, Bart JM, Sako Y, Itoh S, Nakao M, Ito A, Millon L. 2009. Echinococcus vogeli infection in a hunter French Guiana. Emerging Infectious Diseases, 15, 2029-2031.

16. Liance M, Janin V, Bresson-Hadni S, Vuitton DA, Houin R, Piarroux R. 2000. Immunodiagnosis of Echinococcus infections: confirmatory testing and species differentiation by a new commercial Western Blot. Journal of Clinical Microbiology, 38, 3718-3721.

17. Sako Y, Fukuda K, Kobayashi Y, Ito A. 2009. Development of an immunochromatographic test to detect antibodies against recombinant Em18 for diagnosis of alveolar echinococcosis. Journal of Clinical Microbiology, 47, 252-254.
18. Sako Y, Nakao M, Nakaya K, Yamasaki H, Gottstein B, Lightowers MW, Schantz PM, Ito A. 2002. Alveolar echinococcosis: characterization of diagnostic antigen Em18 and serological evaluation of recombinant Em18. Journal of Clinical Microbiology, 40, 2760-2765.

19. Sako Y, Tappe D, Fukuda K, Kobayashi Y, Itoh S, Frosch M, Grüner B, Kern P, Ito A. 2011. Immunochromatographic test with recombinant em18 antigen for the follow-up study of alveolar echinococcosis. Clinical and Vaccine Immunology, 18, 1302-1305.

20. Schweiger A, Ammann RW, Candinas D, Clavien PA, Eckert J, Gottstein B, Halkic N, Muellhaupt B, Prinz BM, Reichen J, Tarr PE, Torgerson PR, Deplazes P. 2007. Human alveolar echinococcosis after fox population increase Switzerland. Emerging Infectious Diseases, 13, 878-882.

21. Thompson RCA, McManus DP. 2002. Aetiology: parasites and life-cycles. Manual WHO/OIE on Echinococcosis in Humans ans Animals: a Public Health Problem of Global Concern, Chapter, 1, 9.

22. Torgerson PR, Keller K, Magnotta M, Ragland N. 2010. The global burden of alveolar echinococcosis. PLoS Neglected Tropical Diseases, 4, e722.

23. van Dommelen L, Stoot JH, Cappendijk VC, Abdul Hamid MA, Stelma FF, Kortbeek LM, van der Giessen J, Oude Lashof AM. 2012. The first locally acquired human infection of Echinococcus multilocularis in the Netherlands. Journal of Clinical Microbiology, 50, 1818-1820.

24. Xiao N, Mamuti W, Yamasaki H, Sako Y, Nakao M, Nakaya K, Gottstein B, Schantz PM, Lightowlers MW, Craig PS, Ito A. 2003. Evaluation of use of recombinant Em18 and affinitypurified Em18 for serological differentiation of alveolar echinococcosis from cystic echinococcosis and other parasitic infections. Journal of Clinical Microbiology, 41, 3351-3353.

Cite this article as: Knapp J, Sako Y, Grenouillet F, Bresson-Hadni S, Richou C, Gbaguidi-Haore H, Ito A \& Millon L: Comparison of the serological tests ICT and ELISA for the diagnosis of alveolar echinococcosis in France. Parasite, $2014,21,34$.

Reviews, articles and short notes may be submitted. Fields include, but are not limited to: general, medical and veterinary parasitology; morphology, including ultrastructure; parasite systematics, including entomology, acarology, helminthology and protistology, and molecular analyses; molecular biology and biochemistry; immunology of parasitic diseases; host-parasite relationships; ecology and life history of parasites; epidemiology; therapeutics; new diagnostic tools.

All papers in Parasite are published in English. Manuscripts should have a broad interest and must not have been published or submitted elsewhere. No limit is imposed on the length of manuscripts.

Parasite (open-access) continues Parasite (print and online editions, 1994-2012) and Annales de Parasitologie Humaine et Comparée (1923-1993) and is the official journal of the Société Française de Parasitologie. 